\title{
Les Rendez-vous de l'histoire de Weimar « Reisen in (Un)Bekanntes/ Destination (in)connue »
}

\author{
Franka Günther \\ Traducteur : Juliette Guilbaud
}

URL : http://journals.openedition.org/ifha/105

DOI : 10.4000/ifha. 105

ISSN : 2198-8943

Éditeur

IFRA - Institut franco-allemand (sciences historiques et sociales)

Édition imprimée

Date de publication : 6 février 2011

Pagination : 16-21

ISSN : 2190-0078

Référence électronique

Franka Günther, «Les Rendez-vous de l'histoire de Weimar « Reisen in (Un)Bekanntes/ Destination (in)connue » », Revue de l'IFHA [En ligne], 3 | 2011, mis en ligne le 01 janvier 2012, consulté le 20 avril 2019. URL : http://journals.openedition.org/ifha/105; DOI : 10.4000/ifha.105

Ce document a été généré automatiquement le 20 avril 2019.

(C)IFHA 


\title{
Les Rendez-vous de l'histoire de Weimar « Reisen in (Un)Bekanntes/ Destination (in)connue »
}

\author{
Franka Günther \\ Traduction : Juliette Guilbaud
}

\section{NOTE DE L'ÉDITEUR}

Weimar, 29-31 octobre 2010

Pour la deuxième année consécutive se tenait ce festival international de l'histoire que sont les Rendez-vous de l'histoire de Weimar, du 29 au 31 octobre 2010. Le thème à l'honneur pour cette édition était « Reisen in (Un)Bekanntes/ Destination (in)connue ». Plus de deux mille visiteurs, toutes générations confondues, se pressèrent aux quelque trente manifestations au programme. Les vingt films projetés dans le cadre du festival de cinéma créé spécialement pour ces Rendez-vous attirèrent près de sept cents spectateurs.

Ces Rendez-vous de Weimar explorèrent les nombreux thèmes suggérés par la notion de «voyage »: comme événement du réel, comme métaphore de la nostalgie (Sehnsucht), comme introspection ou encore comme motif politique. Les discussions et communications proposées lors de ces rencontres internationales furent de haute qualité. De fait, des intervenants en provenance d'Allemagne, de Pologne, de France, de GrandeBretagne, d'Autriche et d'Israël avaient fait le voyage jusqu'à Weimar. Les débats sur des thèmes liés à l'histoire de Weimar suscitèrent tout particulièrement l'intérêt des visiteurs : citons notamment les Rencontres poétiques (Dichtertreffen) de 1941, AnneAmélie de Brunswick (1739-1807, duchesse de Saxe-Weimar-Eisenach à partir de 1756) et les voyages des femmes au XVIIIe siècle, ceux du landgrave de Thuringe en Terre sainte, ceux de Franz Liszt, ou encore Charles-Alexandre de Saxe-Weimar-Eisenach (1818-1901) et son temps. Le public vint en nombre assister au débat sur « Fuite et expulsion », ainsi qu'à la soirée de clôture intitulée "Les repas en voyage ", où l'on put se régaler de plats 
venant des quatre coins de l'Europe et des différentes périodes historiques évoquées. La conférence inaugurale, donnée par Lothar de Maizière, affichait complet avec ses cent quatre-vingts auditeurs. L'ancien ministre-président, premier à avoir été élu librement à ces fonctions à la tête de la RDA et historiquement dernier à occuper ces mêmes fonctions en 1990, évoqua ses expériences de voyage.

Les Rendez-vous de Weimar sont de dimension européenne et centrés sur le Triangle de Weimar (Weimarer Dreieck) qui relie l'Allemagne, la France et la Pologne. Cette caractéristique se reflétait également dans la programmation du festival. En ouverture fut projeté un documentaire sur 1989 et ses bouleversements, suivi d'une table ronde avec Rita Süssmuth, l'ambassadeur de Pologne Marek Prawda et l'ambassadeur de France Bernard de Montferrand. Tous trois livrèrent chacun à l'auditoire, venu nombreux, un regard intéressant sur les événements et les coulisses de l'automne 1989. Il est prévu de développer le Triangle de Weimar dans les années qui viennent, en accentuant encore plus la dimension européenne des Rendez-vous. Pour ce faire, une collaboration encore plus étroite avec les Rendez-vous de l'histoire de Blois, pionniers du genre, est envisagée, ainsi que des échanges de contenus et d'intervenants.

En matière de coopération, les Rendez-vous de Weimar favorisent parmi les visiteurs le mélange des générations. En 2010, pour une part importante de son programme, le festival invitait clairement élèves et étudiants à réfléchir sur des thèmes historiques. À cet égard, la coopération franco-allemande entre les lycées de Weimar et de Beauvais préparant à l'abibac ${ }^{1}$ mérite d'être saluée : les élèves proposèrent une lecture mise en scène de "Correspondances de la Seconde Guerre mondiale ». Grâce au soutien de la chancellerie de Thuringe et de l'office franco-allemand pour la jeunesse, un projet scolaire trilatéral sur les voyages de Chopin et de Liszt a été mis en place. Des élèves de Weimar, Cracovie et Blois organisèrent une exposition sur le thème «Les Artistes, ces migrants, hier et aujourd'hui ». Ces élèves doivent poursuivre leur collaboration à l'avenir : ainsi le projet mis en place sur le thème de "L'Orient »- choisi pour les Rendezvous de l'histoire de Blois en 2011 - sera-t-il également présenté en Pologne, en 2012. Sur le plan académique et universitaire, un colloque des étudiants de Leipzig s'est tenu à Weimar en 2010, tandis que des étudiants d'Erfurt organisaient un séminaire de master et un projet semestriel sur l'histoire de Łódź, en coopération avec l'université de cette ville. Ces actions ont valeur de messages : les Rendez-vous de Weimar sont ouverts à de futures coopérations avec d'autres universités.

Une part importante du programme des Rendez-vous de Weimar, en 2010, consistait en soirées à la fois culinaires et culturelles, occasions pour les quatre importantes communautés de migrants établies à Weimar de dîner et de faire la fête avec les habitants de la ville, tout en livrant un aperçu de leur culture respective. Le succès de ces soirées et la demande encouragent leur poursuite. Pour $2011^{2}$ ont été programmées des discussions avec des habitants de Weimar en provenance d'Israël, de Palestine, de Syrie et d'Iran: occasion pour ces Weimariens d'adoption de livrer une vision personnelle de leur pays d'origine respectif et de répondre aux questions sur les sujets brûlants qui les concernent. Et la soirée de se poursuivre, pour l'ensemble des participants, par de la musique et de la danse.

Le concept du festival d'histoire de Weimar - être un vecteur des questions historiques et politiques, maintenir un niveau scientifique exigeant, mais sans abuser du jargon scientifique - est accueilli favorablement par un public de plus en plus large, et peut donc être considéré comme une réussite. Le bilan est très positif aux yeux du public, comme en 
témoigne un sondage réalisé auprès des visiteurs. La manifestation est «bien arrivée à Weimar " et les efforts des organisateurs visent à fidéliser le public, notamment en intégrant au projet de nouvelles écoles et universités, des enseignants, ou encore en activant différents réseaux. Les institutions reçoivent «carte blanche » et peuvent ainsi organiser leurs propres manifestations sur le thème du festival.

Programme des conférences en $2010^{3}$ :

\begin{tabular}{|c|c|}
\hline Thèmes & Intervenants \\
\hline Conférence inaugurale & $\begin{array}{l}\text { Lothar de Maizière (ancien ministre-président } \\
\text { et ancien ministre fédéral) }\end{array}$ \\
\hline En coulisse. La diplomatie en 1989 & $\begin{array}{l}\text { Bernard de Montferrand (ambassadeur de } \\
\text { France), Marek Prawda (ambassadeur de } \\
\text { Pologne), Rita Süssmuth (ancienne présidente } \\
\text { du Bundestag) }\end{array}$ \\
\hline $\begin{array}{l}\text { Comme un voyage vers les mers du Sud. Les } \\
\text { récits de voyageurs allemands à Majorque au } \\
\text { XIXe siècle }\end{array}$ & Ekkehard Schönherr (Erfurt) \\
\hline $\begin{array}{l}\text { L'appel du Sud. Les voyages vers l'Italie, du } \\
\text { furor Teutonicus à la «fraction toscane" }\end{array}$ & Helmut Walther (Iéna) \\
\hline $\begin{array}{l}\text { Fuite ou expulsion? Quand la politique se } \\
\text { mêle au débat historique... }\end{array}$ & $\begin{array}{l}\text { Stefan Troebst (Leipzig), Krzysztof Ruchniewicz } \\
\text { (Wrocław), Alice Volkwein (Paris/ Munich). } \\
\text { Modération : Claudia Kraft (Erfurt) }\end{array}$ \\
\hline $\begin{array}{l}\text { Terra Sancta. Des Thuringiens en route vers } \\
\text { la Palestine }\end{array}$ & Marc Delpech (Weimar) \\
\hline $\begin{array}{l}\text { De l'Allemagne vers l'Europe en passant par } \\
\text { Le Rhin, ou comment la vision du voyage s'est } \\
\text { transformée du XIXe au XXe siècle (à partir } \\
\text { de textes de Mme de Staël, Victor Hugo et le } \\
\text { comte Kessler) }\end{array}$ & Annette Seemann (Weimar) \\
\hline $\begin{array}{l}\text { La Florence des bords de l'Ilm mise en scène. } \\
\text { Artistes, rendez-vous artistiques et image de } \\
\text { Weimar au temps de Charles-Alexandre }\end{array}$ & Alf Rößner (Weimar) \\
\hline $\begin{array}{l}\text { Frontières nationales et mobilité nouvelle en } \\
\text { Europe }\end{array}$ & $\begin{array}{l}\text { Nicolas Beaupré (Clermont-Ferrand), Bernhard } \\
\text { Struck (St. Andrews), Frithjof Benjamin Schenk } \\
\text { (Munich). Modération: Étienne François } \\
\text { (Berlin) }\end{array}$ \\
\hline $\begin{array}{l}\text { Femmes en voyage. Les espaces de } \\
\text { mouvement féminins au XVIIIe siècle }\end{array}$ & $\begin{array}{l}\text { Friederike Kuster (Siegen), Irmgard Scheitler } \\
\text { (Wurtzbourg), Annegret Pelz (Vienne), Siegfried } \\
\text { Seifert (Weimar). Modération: Justus H. } \\
\text { Ulbricht (Dresde/ Weimar) }\end{array}$ \\
\hline
\end{tabular}




\begin{tabular}{|c|c|}
\hline $\begin{array}{l}\text { Le dernier - ou le grand - voyage de Maurice } \\
\text { Halbwachs: Buchenwald }\end{array}$ & Annette Becker (Paris) \\
\hline Voyager pour la paix, du Moyen Âge à 1945 & $\begin{array}{l}\text { Frank Estelmann (Francfort/Main), Stéphane } \\
\text { Péquignot (Paris), Anuschka Tischer (Marburg), } \\
\text { Olaf Müller (Iéna). Modération: Nicolas } \\
\text { Offenstadt (Paris) }\end{array}$ \\
\hline Anne-Amélie en Italie & $\begin{array}{l}\text { Siegfried Seifert (Weimar), Thomas Kroll (Iéna), } \\
\text { Klaus Manger (Iéna) }\end{array}$ \\
\hline Les Rencontres poétiques de Weimar en 1941 & $\begin{array}{l}\text { Étienne François (Berlin), Anne Kwaschik } \\
\text { (Berlin), Justus H. Ulbricht (Dresde/ Weimar). } \\
\text { Modération : Johann Chapoutot (Grenoble) }\end{array}$ \\
\hline $\begin{array}{l}\text { Les voyages de Jean-Paul II et leur sens pour } \\
\text { l'histoire }\end{array}$ & Theo Mechtenberg (Bad Oeynhausen) \\
\hline $\begin{array}{l}\text { Des Chinois à Hambourg, Londres ou } \\
\text { Rotterdam : une histoire globale et locale des } \\
\text { migrations }\end{array}$ & Lars Amenda (Osnabrück) \\
\hline I love Liszt. Les voyages d'un citoyen du monde & Nicolas Dufetel (Weimar/ Paris) \\
\hline $\begin{array}{l}\text { L'Europe-forteresse ou la liberté à l'intérieur } \\
\text { de frontières }\end{array}$ & 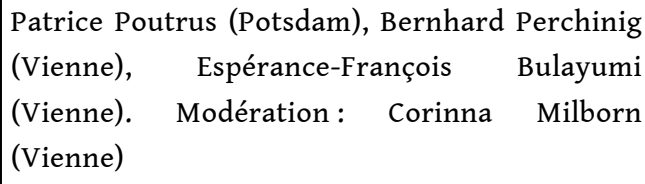 \\
\hline $\begin{array}{l}\text { De Weimar jusqu'en Afrique : Gerhard Rohlfs } \\
\text { et les recherches allemandes sur l'Afrique au } \\
\text { XIXe siècle }\end{array}$ & Aurélia Dusserre (Aix-Marseille) \\
\hline $\begin{array}{l}\text { L'Allemagne à genoux : la réconciliation après } \\
1945\end{array}$ & $\begin{array}{l}\text { Hélène Miard-Delacroix (Paris), Krzysztof } \\
\text { Ruchniewicz (Wrocław), Moshe Zimmermann } \\
\text { (Jérusalem). Modération: Thomas Serrier } \\
\text { (Francfort/Oder) }\end{array}$ \\
\hline $\begin{array}{l}\text { "Nous, nous partons; nous, nous restons." } \\
\text { Le voyage comme motif politique dans } \\
\text { l'ancien bloc de l'Est }\end{array}$ & $\begin{array}{l}\text { Ilko-Sascha Kowalczuk (Berlin), Claudia Müller } \\
\text { (Leeds), Christian Halbrock (Stasi-Unterlagen } \\
\text { Behörde). Modération: Martin Sabrow } \\
\text { (Potsdam/Berlin) }\end{array}$ \\
\hline $\begin{array}{l}\text { Aller-retour de l'« autre côté " (à partir des } \\
\text { contes des frères Grimm et d'Alexandre } \\
\text { Afanassiev) }\end{array}$ & Natacha Rimasson-Fertin (Grenoble) \\
\hline $\begin{array}{l}\text { De la Hanse au programme Erasmus: } \\
\text { l'édification de l'Europe }\end{array}$ & $\begin{array}{l}\text { Daniel Schönpflug (Berlin), Andreas Ranft } \\
\text { (Halle-Wittenberg), Anne-Marie Autissier } \\
\text { (Paris). Modération: Thomas Serrier } \\
\text { (Francfort/Oder) }\end{array}$ \\
\hline
\end{tabular}




\section{NOTES}

1. Diplôme binational équivalent à la fois du baccalauréat français et de l'Abitur allemand (NdT).

2. Le thème de l'édition 2011 des Rendez-vous de l'histoire de Weimar (4-6 novembre 2011), est : «Gewaltmenschen. Menschengewalt / Homme(s) et violence(s)».

3. À retrouver en allemand dans les archives du site des Rendez-vous de l'histoire de Weimar : http://www.weimarer-rendezvous.de/archiv/archiv2010.html. 